\title{
Desigualdad y polarización social en comunas de Chile
}

\author{
Jessica Candia Cid, José Merino Escobar, \\ Claudio Bustos y David Martínez
}

\section{Resumen}

La desigualdad social producto de la actual distribución de los ingresos en Chile mantiene el interés por conocer sus tendencias en diversos contextos. El propósito de este trabajo fue analizar, a nivel comunal, la desigualdad y la polarización como consecuencia de la distribución de los ingresos, utilizando los ingresos autónomos y totales per cápita. Se analizaron 78 comunas pertenecientes a 5 regiones del norte, centro y sur de Chile. Los resultados confirman una significativa desigualdad en la distribución de los ingresos y una marcada polarización a nivel comunal. Estos hallazgos justifican la necesidad de crear políticas de redistribución de ingresos focalizadas y pertinentes a nivel de comunas, ya que la desigualdad y la polarización se han vinculado con sentimientos de malestar en la población producto de las situaciones de conflictos e injusticias sociales que se generan y potencian.

\section{Palabras clave}

Condiciones económicas, condiciones sociales, distribución del ingreso, igualdad, pobreza, conflicto social, análisis de datos, indicadores económicos, Chile

\section{Clasificación JEL}

D31, D63

\section{Autores}

Jessica Candia, Departamento de Psiquiatría y Salud Mental de la Universidad de Concepción y Facultad de Psicología de la Universidad San Sebastián, Chile. Correo electrónico: jecandia@udec.cl.

José Merino Escobar, Facultad de Sociología de la Universidad de Concepción, Chile. Correo electrónico: jmerino@udec.cl.

Claudio Bustos, Facultad de Medicina de la Universidad de Concepción, Chile. Correo electrónico: clbustos@udec.cl.

David Martínez, Facultad de Psicología de la Universidad San Sebastián, Chile. Correo electrónico: david.martinez@uss.cl. 


\section{Introducción}

La reducción de las desigualdades forma parte de uno de los 17 Objetivos de Desarrollo Sostenible (ODS) acordados por los países del mundo en la Agenda 2030 para el Desarrollo Sostenible, que la Asamblea General de las Naciones Unidas aprobó en 2015 (PNUD, 2017). En algunos estudios se ha argumentado la necesidad de que las significativas y persistentes desigualdades sociales presentes en el mundo sean abordadas a través de políticas sociales dirigidas a su disminución (Alvaredo y otros, 2018). Por su parte, la distribución de los ingresos en una sociedad, en materia de igualdad o desigualdad, constituye un tema fundamental y actualmente de interés (Wilkinson, 2005; Uribe López, 2009; Amarante y Colacce, 2018), debido a la vasta información empírica existente acerca del vínculo entre las brechas en el ingreso y ciertos efectos psicosociales negativos importantes en las personas (Wilkinson y Pickett, 2009; Campos-Arias y Herazo, 2015; Quijada y otros, 2018; Navarro Yánez y Pérez Yruela, 2000) y sentimientos de malestar (PNUD, 2017), aunque se reconoce que la relación existente entre la desigualdad social producto de la distribución de los recursos y el malestar experimentado por las personas puede estar mediada o moderada por otras variables sociales. Así también, a nivel macro se ha evidenciado que el estigma que la desigualdad produce se percibe negativamente en diferentes ámbitos como el financiero, material, de capacidades, de oportunidades, de acceso al bienestar, de relaciones sociales y de respeto a los derechos de las personas, ya que la desigualdad conlleva un desaprovechamiento de las capacidades de las personas, deslegitima la política y afecta la democracia y la convivencia, favoreciendo la generación de conflictos (CEPAL, 2018).

En las últimas décadas, con el propósito de lograr una mayor comprensión de la desigualdad social y sus efectos en las personas y la sociedad, se ha incorporado el concepto de polarización para explicar el nivel de conflictividad producto de la desigualdad (Wolfson, 1997; Esteban y Ray, 1994). Aunque la desigualdad y la polarización son fenómenos sociales relacionados, la polarización considera un aspecto que la desigualdad no incluye; a saber, permite mostrar en qué medida la población está agrupada en torno a un pequeño número de polos o conglomerados, internamente homogéneos y heterogéneos entre sí, que estarían potenciando la generación de tensiones sociales (Vergara, 2011). Además, se ha asociado a un sentimiento de malestar en las personas. Recientemente, en un estudio realizado en España se concluyó que los niveles de satisfacción como resultado de las mejoras de las condiciones de vida de la población esconden, e incluso pueden potenciar, una sociedad polarizada entre un grupo de ciudadanos con carencias y necesidades pero que se manifiestan satisfechos, y otro grupo de ciudadanos en situación de bienestar, satisfechos consigo mismos, pero no con el Estado de bienestar ni con la sociedad en la que viven (Navarro Yánez y Pérez Yruela, 2000).

Es destacable que no son pocos los estudios que se han llevado adelante con el interés de demostrar la alta desigualdad y la polarización social presentes en Chile, en la mayoría de los cuales se han realizado análisis y comparaciones a nivel de país y, en algunos casos, de región (Contreras, 1999; Schatan, 2005; Raventós, 2005; Conte, 2008; Vergara, 2011; Silva Burgos, 2013; PNUD, 2017). En menor medida, se encuentran algunas investigaciones sobre la desigualdad a nivel comunal, en que se han utilizado datos de la Encuesta de Caracterización Socioeconómica Nacional (CASEN) (1992-2003) y de los censos (Ruiz-Tagle, 1999; Vergara, 2011). Uno de ellos muestra cómo la desigualdad a nivel comunal dificulta la reducción de la pobreza y además influye en el surgimiento de problemas sociales que afectan el desarrollo del país (Agostini y Brown, 2007). A su vez, otra investigación revela la existencia de zonas de mejor y de peor distribución del ingreso y que las dinámicas de distribución no han sido regulares, al menos desde la década de 1990 en adelante (Ramírez, Tartakowsky y Modrego, 2009). Por su parte, Carpentier (2011) examina la desigualdad a nivel comunal considerando variables asociadas a la educación. Es así como concluye que la escolaridad promedio y la participación laboral femenina tienen un efecto reductor de la desigualdad de ingresos a nivel comunal.

Cabe señalar que, a pesar de la existencia de esta literatura, no se dispone de información concluyente respecto a cómo se presenta el fenómeno de la desigualdad vinculado a la polarización 
social a niveles territoriales más desagregados. En ese contexto, este artículo está orientado por la siguiente pregunta: ¿cómo se presentan la desigualdad y la polarización social producto de la distribución de los ingresos a nivel de comunas en Chile? A partir de esta pregunta, el supuesto es que la persistente y marcada desigualdad que se ha evidenciado a nivel regional y nacional se mantiene e incluso se exacerba en algunas comunas, generando territorios polarizados. Esto se evidencia en la presencia de unos pocos grupos altamente concentrados y homogéneos internamente y con gran distanciamiento social entre sí.

Para abordar la pregunta de investigación de este trabajo se plantearon los siguientes objetivos: i) analizar la desigualdad en la distribución de los ingresos en las comunas y ii) examinar la polarización social producto de la distribución de los ingresos en las comunas.

Para desarrollar los puntos antes descritos, el artículo posee la siguiente estructura. En primer lugar, se exponen los principales avances sociales de Chile desde la década de 1990, como resultado de las políticas sociales y del crecimiento económico, a fin de situar y comprender los escasos progresos en materia de igualdad o desigualdad social producto de la distribución de los ingresos (sección II). En segundo lugar, se presentan antecedentes conceptuales del coeficiente de Gini como medida de la desigualdad y del índice de polarización, con el que busca identificar la presencia de polos y las distancias existentes entre ellos como consecuencia de la distribución de los ingresos (sección III). En tercer lugar, se describe la metodología utilizada para el análisis de la desigualdad y la polarización de 78 comunas de 5 regiones del país (sección IV). En cuarto lugar, se presentan los resultados de esta investigación, que confirman una significativa desigualdad en la distribución de los ingresos y una marcada polarización a nivel comunal (sección V). Finalmente, se realiza un análisis de la comparación entre el coeficiente de Gini con ingreso per cápita autónomo y el coeficiente de Gini con ingreso per cápita total, que destaca el efecto aún insuficiente de las transferencias monetarias en la reducción de la desigualdad y la necesidad de establecer políticas públicas dirigidas específicamente a su abordaje a nivel comunal (sección VI).

\section{Antecedentes de la política social en Chile y sus principales avances}

Desde los años noventa, después de la dictadura militar, se inició en Chile la instalación de un modelo de desarrollo "con equidad", con el que se buscó superar los altos índices de pobreza del país, a través de la apertura a los mercados internacionales y del incremento progresivo del gasto social ${ }^{1}$ público (Cleary, 2007), que aumentó de un 11,6\% del producto interno bruto (PIB) en 2011 a un 13,9\% en 2016 (OCDE, 2018). Lo anterior produjo efectivamente un impacto en los indicadores de pobreza, que pasó de un 22,2\% (14,1\% de pobreza no extrema y 8,1\% de pobreza extrema) en 2011 a un 8,6\% (6,3\% de pobreza no extrema y 2,3\% de pobreza extrema) en 2017 (Ministerio de Desarrollo Social, 2018). Además, se introdujeron reformas en el sistema tributario para dotarlo de mayor progresividad, expandiendo la oferta de programas sociales. Con estas reformas se buscaban, además, mejoras en indicadores indirectos de la desigualdad, como el cierre de las brechas salariales entre hombres y mujeres. Sin embargo, la dualidad del trato entre géneros del mercado laboral mantiene una distribución de salarios muy desigual (OCDE, 2015).

Fue así como el modelo de desarrollo generó períodos de crecimiento económico sobre el $7 \%$ del PIB ${ }^{2}$, rebajas de la inflación y reducción de la deuda pública y externa, y permitió evitar los

\footnotetext{
1 En el gasto social se considera el gasto en educación, salud y protección social (OCDE, 2018).

2 El PIB a precio de comprador es la suma del valor agregado bruto de todos los productos residentes en la economía más todo impuesto a los productos, menos subsidios, en un tiempo determinado, y se puede considerar como el poder económico de un país.
} 
efectos negativos de las crisis macroeconómicas, convirtiendo al país en un mercado de interés para inversiones extranjeras. Igualmente, se lograron mejoras en algunos indicadores en los que se alcanzaron niveles de países desarrollados, como el aumento de la cobertura en educación básica y media y de los años de escolaridad obligatoria; el aumento de la esperanza de vida, las tasas de alfabetización y los niveles nutricionales, y se redujeron las tasas de mortalidad materno-infantil (Araya Rosales y Gallardo Altamirano, 2015; CEPAL, 2017). Sin embargo, aun cuando se logró un alto crecimiento económico, se mantuvieron elevados indicadores de desigualdad, que a la vez han ido generando otras limitaciones sociales y reduciendo las posibilidades de las personas de ascender en la escala social (OCDE, 2015).

Lo anterior revela que las políticas sociales no han apuntado a la reducción de la brecha de desigualdad existente, de manera que, si bien hay algunos mecanismos que apuntan indirectamente a este propósito, como las políticas de transferencias monetarias dirigidas a la población en situación de pobreza fundamentalmente por ingresos, estos no constituyen una herramienta de acción estatal de redistribución de la riqueza propiamente tal (Pizarro, 2005; OCDE, 2014; Araya Rosales y Gallardo Altamirano, 2015). Esto queda aún más en evidencia si se compara el coeficiente de Gini de Chile con el de los países con los que comparte el hecho de estar entre los que presentan ingresos altos y ser miembro de la Organización de Cooperación y Desarrollo Económicos (OCDE) (véase el cuadro 1). El coeficiente de Gini de Chile ha estado permanentemente más cerca de aquellos de los países de ingresos bajos y medios, lo que demuestra que ha aumentado la riqueza ${ }^{3}$, pero no se han generado mecanismos para una mejor distribución del ingreso (OCDE, 2014; Vivanco Muñoz y otros, 2015).

\section{Cuadro 1}

Coeficiente de Gini de países seleccionados, según su nivel de ingreso

\begin{tabular}{|c|c|c|c|c|c|c|c|c|}
\hline $\begin{array}{l}\text { Países de ingresos altos y } \\
\text { miembros de la Organización } \\
\text { de Cooperación y Desarrollo } \\
\text { Económicos (OCDE) }\end{array}$ & $\begin{array}{l}\text { Coeficiente } \\
\text { de Gini }\end{array}$ & Año & $\begin{array}{l}\text { Países de ingresos } \\
\text { medio altos y } \\
\text { medio bajos }\end{array}$ & $\begin{array}{l}\text { Coeficiente } \\
\text { de Gini }\end{array}$ & Año & $\begin{array}{l}\text { Países de } \\
\text { ingresos } \\
\text { bajos }\end{array}$ & $\begin{array}{l}\text { Coeficiente } \\
\text { de Gini }\end{array}$ & Año \\
\hline Austria & 0,31 & 2014 & Armenia & 0,32 & 2015 & Benin & 0,48 & 2015 \\
\hline Bélgica & 0,28 & 2014 & Belarús & 0,27 & 2015 & Burundi & 0,39 & 2013 \\
\hline Chile & 0,48 & 2015 & $\begin{array}{l}\text { Bolivia (Estado } \\
\text { Plurinacional) }\end{array}$ & 0,46 & 2015 & Comoras & 0,45 & 2013 \\
\hline Chequia & 0,26 & 2014 & Brasil & 0,51 & 2015 & Madagascar & 0,43 & 2012 \\
\hline Dinamarca & 0,29 & 2014 & Colombia & 0,51 & 2015 & Níger & 0,34 & 2014 \\
\hline España & 0,36 & 2014 & Costa Rica & 0,48 & 2015 & Rwanda & 0,50 & 2013 \\
\hline Eslovenia & 0,26 & 2014 & Ecuador & 0,47 & 2015 & Togo & 0,43 & 2015 \\
\hline Finlandia & 0,27 & 2014 & Egipto & 0,32 & 2015 & & & \\
\hline Italia & 0,35 & 2014 & El Salvador & 0,41 & 2015 & & & \\
\hline Islandia & 0,26 & 2014 & Filipinas & 0,40 & 2015 & & & \\
\hline Noruega & 0,27 & 2014 & Honduras & 0,50 & 2015 & & & \\
\hline Suecia & 0,27 & 2014 & Panamá & 0,51 & 2015 & & & \\
\hline \multirow[t]{2}{*}{ Uruguaya $^{a}$} & 0,42 & 2015 & Paraguay & 0,48 & 2015 & & & \\
\hline & & & Perú & 0,44 & 2015 & & & \\
\hline
\end{tabular}

Fuente: Elaboración propia, sobre la base de datos de Banco Mundial, "Indicadores", s/f [en línea] https://datos.bancomundial. org/indicador.

Nota: El Banco Mundial genera una clasificación de los países según su ingreso nacional bruto (INB) per cápita (alto, medio alto, medio bajo, bajo), usando el método Atlas. Para los umbrales se utiliza el dólar. Véase más información respecto de los umbrales en Banco Mundial, Banco Mundial Blogs, s/f [en línea] https://blogs.worldbank.org/es.

a El Uruguay no es miembro de la OCDE.

3 El PIB de Chile ha presentado un aumento sistemático: pasó de ( 33,11 miles de millones de dólares en 1990 a 77,86 miles de millones de dólares en 2000, 218,53 miles de millones de dólares en 2010 y 250,03 miles de millones de dólares en 2016 (Banco Mundial, s/f). 
Como lo indican los datos disponibles, el promedio del coeficiente de Gini de los países miembros de la OCDE es de 0,30, con un rango de entre 0,27 y 0,48; el 67\% de los 12 países considerados se encuentran bajo el promedio, en tanto que Chile presenta el mayor coeficiente de Gini (véase el cuadro 1).

Pizarro (2005) plantea que se ha generado un repliegue del Estado de bienestar, que se ha traducido en una disminución de los sistemas de protección social y las aspiraciones de igualdad de oportunidades, dado que la regulación del Estado hacia el mercado se ha debilitado y ha dejado que el modelo económico capitalista se autorregule. Debido a lo anterior, el Estado ha quedado relegado a una actividad subsidiaria, con recursos focalizados en los hogares de los primeros quintiles, a través de las políticas de transferencias monetarias. Sin embargo, el crecimiento económico del país y sus beneficios no han apuntado a disminuir la desigualdad a niveles aceptables, sino que la desigualdad ha permanecido con variaciones poco relevantes - con un coeficiente de Gini calculado con el ingreso autónomo de 0,49 y uno calculado con el ingreso monetario de 0,48 en 2015 (Ministerio de Desarrollo Social, 2017) - , lo que mantiene a Chile entre los países más desiguales del mundo (Solimano y Torche, 2008) (véase el cuadro 2).

Cuadro 2

Chile: evolución del coeficiente de Gini, 2006-2015

\begin{tabular}{llllll}
\hline & 2006 & 2009 & 2011 & 2013 & 2015 \\
\hline Coeficiente de Gini con ingreso autónomo (GPA) & 0,50 & 0,51 & 0,50 & 0,50 & 0,49 \\
\hline Coeficiente de Gini con ingreso monetario (GPT) & 0,49 & 0,49 & 0,49 & 0,49 & 0,48 \\
\hline
\end{tabular}

Fuente: Elaboración propia, sobre la base de Ministerio de Desarrollo Social, Informe de Desarrollo Social 2017, Santiago, 2017 [en línea] http://www.desarrollosocialyfamilia.gob.cl/storage/docs/Informe_de_Desarrollo_Social_2017.pdf.

\section{Antecedentes del coeficiente de Gini y polarización social}

La desigualdad en la distribución de los ingresos ha dado origen a variados estudios que han utilizado, entre otros, el coeficiente de Gini para medirla y comprobar su asociación con diversos problemas sociales (Kennedy, Kawachi y Prothrow-Stith, 1996; Nagel, 1974; Vergara, 2011; Campos-Arias y Herazo, 2015; Gatica y otros, 2017). Los estudios, además, han contribuido a identificar y comprender algunos contextos en los que la desigualdad social emerge y se potencia (Schatan, 2005; Silva Burgos, 2013).

El coeficiente de Gini expresa las diferencias de ingreso que se dan entre todos los individuos y suma todas las diferencias absolutas. Toma valores entre 0 y 1: un valor que tiende a 1 refleja una mayor desigualdad y otro que tiende a 0 una mayor igualdad en la distribución del ingreso (Esteban y Ray, 1994).

De acuerdo con Pigaou-Dalton (Vergara, 2011), las medidas de desigualdad deben cumplir con la propiedad básica del principio de transferencia, según el cual una transferencia de un individuo a otro con menos recursos reduce la desigualdad. Por consiguiente, la desigualdad económica resulta del grado de dispersión de los ingresos respecto de un valor de referencia, el ingreso promedio, que describe una situación de perfecta igualdad, en la que todos tendrían los mismos ingresos. Para medirla se emplean diversos índices que presentan diferentes sensibilidades a las transferencias que se producen en los distintos puntos de la distribución. El coeficiente de Gini presenta una mayor sensibilidad a las transferencias que se producen en el centro de la distribución.

Si bien existe consenso respecto a que la utilización de este índice presenta limitaciones (Escobar, 1998; Contreras, 1999; Ortiz y Cummins, 2011), algunas de ellas no son de responsabilidad exclusiva del estadístico, sino que más bien son producto de la diversidad y la calidad de los datos de ingresos de hogares con los que se calcula, tanto a niveles locales como para la comparación internacional. 
Pero también los autores plantean que el coeficiente de Gini es un indicador que presenta mayor poder predictivo y que su uso puede ser complementado con otros indicadores (índice de Palma, índice de Theil, razón de quintiles y coeficiente de Atkinson, entre los más usados) que permitan realizar un análisis más complejo del comportamiento de la distribución de los ingresos en una sociedad.

Por su parte, el índice de polarización ha sido utilizado para entender el impacto negativo que tiene la distribución o concentración de los recursos de una sociedad (Duclos, Esteban y Ray, 2004; Cárdenas, 2011; Villalobos y Valenzuela, 2012) y permite explicar algunas asociaciones entre la distribución de los recursos y la generación de polos, que corresponden a conjuntos o grupos de personas u hogares, muy heterogéneos entre sí, pero internamente muy homogéneos (por ejemplo, si se observan los hábitos de consumo por quintiles de pertenencia, se advertirá que quienes pertenecen al quintil I comparten internamente algunas preferencias de productos, sistemas de pago y lugares de compra diferentes de aquellos de quienes pertenecen al quintil $V$, que presentan otras preferencias). La existencia de tales polos posibilita el surgimiento de conflictos sociales debido a la distancia social que se genera entre ellos (Gradín y Rossi, 2002; Cárdenas, 2011; Huesca Reynoso, 2003; Villalobos y Valenzuela, 2012).

En el presente análisis se utilizó la ecuación para el cálculo de la polarización propuesta por Duclos, Esteban y Ray (2004), valorando que este no requiere tener definidos a priori los conglomerados de pertenencia de cada uno de los casos en análisis, como se requiere en otros métodos. Por lo tanto, este indicador utiliza la distribución continua de ingresos para estimar el grado de probabilidad de que una persona presente similar ingreso respecto de otras. Es así como, si el ingreso de un caso es igualmente probable que el ingreso de otro caso, se presenta una polarización igual al coeficiente de Gini (distribución uniforme); pero cuando se presentan grupos de ingresos diferentes, ampliamente separados entre sí, se genera un aumento de la polarización, aun cuando el coeficiente de Gini puede ser igual.

En términos teóricos, el mínimo de polarización es 0 (esto se presenta cuando el coeficiente de Gini es 0) y su máximo teórico es infinito. Hay que tener en cuenta que el cálculo de la polarización depende del valor de Alfa seleccionado. Un valor de Alfa pequeño redunda en que los valores de polarización sean más cercanos al coeficiente de Gini, en tanto que valores de Alfa mayores (cercanos a 1) tienden a extremar las diferencias de polarización al mismo tamaño de brechas.

La fórmula de la polarización es la siguiente"

$$
P_{\alpha}(f) \equiv \iint f(x)^{1+\alpha} f(y)|y-x| d y d x
$$

donde $\propto \in[0,25 ; 1]$.

De acuerdo con Esteban y Ray (1994), la polarización aumenta con la heterogeneidad entre conglomerados, con la homogeneidad intraconglomerados y cuanto menor es el número de conglomerados con mayor número de integrantes. Las dos últimas características marcan la diferencia con la desigualdad, ya que cuanto mayor es la homogeneidad en los conglomerados más decrece la desigualdad y crece la polarización (Huesca Reynoso, 2003; Conte, 2008; Vergara, 2011), debido a que en la polarización los individuos aislados tienen un menor peso que en los indicadores para medir la desigualdad y para que esta aumente se necesita la mayor heterogeneidad entre todas las observaciones.

4 Duclos, Esteban y Ray (2004). 


\section{Metodología}

La muestra estuvo compuesta por un total de 78 comunas $^{5}$, correspondientes a un $23 \%$ del número total de comunas, pertenecientes a 5 regiones que representan al norte, centro y sur del país 6 . El tamaño de la muestra considera un $n$ de entre el 33,3\% y el 50\% del total de comunas por cada región: en la Región de Coquimbo, 5 de un total de 15; en la de Valparaíso, 13 de un total de 38; en la del Biobío, 19 de un total de 54; en la de La Araucanía, 15 de un total de 32, y en la Metropolitana, 26 de un total de 52. Para los cálculos del coeficiente de Gini se utilizaron el ingreso autónomo per cápita ${ }^{7}$ y el ingreso total per cápita ${ }^{8}$, y se aplicó el factor de expansión comunal ${ }^{9}$.

El análisis de los datos se realizó en cuatro momentos. En un primer momento se calcularon los siguientes índices: coeficientes de Gini con ingreso per cápita autónomo (GPA) ${ }^{10}$ y de Gini con ingreso per cápita total (GPT) ${ }^{11}$ e índices de polarización con ingreso per cápita autónomo (PPA) ${ }^{12}$ y de polarización con ingreso per cápita total (PPT) $)^{13}$ de las 78 comunas analizadas. Para el cálculo de la polarización se probaron distintos valores de Alfa, y se observó, al usar la técnica de bootstrap, un importante sesgo con Alfas mayores que 0,5 en comunas con Gini alto, como Traiguén, por lo que se decidió usar Alfa 0,25. Es importante destacar que el orden de los casos (que en este estudio corresponde a comunas), basado en el cálculo de su polarización, es independiente del Alfa seleccionado, ya que se mantiene la jerarquía independientemente de este. Luego, en un segundo momento, se realizó la prueba de Student para muestras dependientes, a fin de establecer las diferencias entre el GPA y el GPT, y entre el PPA y el PPT. En un tercer momento, se realizaron análisis descriptivos para conocer el comportamiento y las tendencias de las variables analizadas en las 78 comunas. Finalmente, se realizó una correlación de Pearson para determinar la relación entre desigualdad y polarización (véase el cuadro 3).

\section{Cuadro 3}

Correlación de Pearson entre el coeficiente de Gini y el índice de polarización de las 78 comunas analizadas

\begin{tabular}{lcc}
\hline & $\begin{array}{c}\text { Polarización con ingreso } \\
\text { per cápita autónomo }\end{array}$ & $\begin{array}{c}\text { Polarización con ingreso } \\
\text { per cápita total }\end{array}$ \\
\hline Gini con ingreso per cápita autónomo & $0,77^{\star *}$ & $0,73^{\star *}$ \\
\hline Gini con ingreso per cápita total & $0,81^{\star *}$ & $0,81^{\star *}$ \\
\hline
\end{tabular}

Fuente: Elaboración propia.

Nota: $\quad{ }^{\star}: p<0,05 ;{ }^{* \star}: p<0,01$.

5 Las comunas escogidas por cada región corresponden a todas las que cuentan con representatividad comunal en la CASEN 2015.

6 En la CASEN 2015 se realiza un análisis con las 15 regiones del país existentes en ese momento. Posteriormente, el 12 de julio de 2017, se creó la XVI Región de Ñuble, a partir de la división de la Región del Biobío.

7 El ingreso autónomo per cápita del hogar corresponde a la suma de todos los pagos que reciben las personas del hogar, provenientes del trabajo, la propiedad y los activos, incluidos sueldos y salarios, monetarios y en especies, ganancias del trabajo independiente, la autoprovisión de bienes producidos por el hogar, rentas, intereses, dividendos y retiro de utilidades, jubilaciones, pensiones o montepíos, y transferencias corrientes, dividida por el número de miembros del hogar.

8 El ingreso total per cápita del hogar corresponde al ingreso autónomo más subsidios monetarios que reciben todas las personas del hogar, excluido el servicio doméstico puertas adentro, dividido por el número de miembros del hogar.

9 El factor de expansión corresponde a una medida estadística que permite expandir cada contribución de la observación muestral para reflejar su participación en la población del estudio.

10 Para el cálculo del coeficiente de Gini con ingreso per cápita autónomo (GPA) se utiliza el ingreso autónomo per cápita de un hogar.

${ }^{11}$ Para el cálculo del coeficiente de Gini con ingreso per cápita total (GPT) se utiliza el ingreso total per cápita de un hogar.

12 Para el cálculo del índice de polarización con ingreso per cápita autónomo (PPA) se utiliza el ingreso autónomo per cápita de un hogar.

${ }^{13}$ Para el cálculo del índice de polarización con ingreso per cápita total (PPT) se utiliza el ingreso total per cápita de un hogar. 


\section{Resultados}

\section{Cálculo y análisis de los coeficientes de Gini e índices de polarización de las 78 comunas}

En el análisis del coeficiente de Gini de las comunas se evidencia una gran variabilidad, con un GPA de entre de 0,36 y 0,81 (con una media $(M)$ de 0,46 y una desviación estándar ( $D E$ ) de 0,06) y un GPT de entre 0,29 y 0,74 (M 0,39; DE 0,06) (véase el cuadro 4). La comuna que presenta menor desigualdad es Cerro Navia, de la Región Metropolitana de Santiago, y la que presenta mayor desigualdad Traiguén, de la Región de La Araucanía.

\section{Cuadro 4}

Estadísticos descriptivos: coeficiente de Gini e índice de polarización de las 78 comunas analizadas

\begin{tabular}{lcccccc}
\hline & N & Mínimo & Máximo & Media & Mediana & $\begin{array}{c}\text { Desviación } \\
\text { estándar }\end{array}$ \\
\hline Gini con ingreso per cápita autónomo (GPA) & 78 & 0,36 & 0,81 & 0,46 & 0,45 & 0,06 \\
\hline Gini con ingreso per cápita total (GPT) & 78 & 0,29 & 0,74 & 0,39 & 0,38 & 0,06 \\
\hline Polarización con ingreso per cápita autónomo (PPA) & 78 & 0,15 & 5,05 & 0,25 & 0,19 & 0,55 \\
\hline Polarización con ingreso per cápita total (PPT) & 78 & 0,15 & 4,18 & 0,24 & 0,19 & 0,45 \\
\hline
\end{tabular}

Fuente: Elaboración propia, sobre la base de datos de Ministerio de Desarrollo Social y Familia, "Encuesta CASEN 2015", Observatorio Social, Santiago [en línea] http://observatorio.ministeriodesarrollosocial.gob.cl/casen-multidimensional/ casen/casen_2015.php.

En cuanto al índice de polarización, en el que se observa una gran variabilidad, mayor que la observada en el coeficiente de Gini, el PPA se encuentra entre 0,15 y 5,05 (M 0,19; DE 0,55) y el PPT entre 0,15 y 4,18 (M 0,24; DE 0,45). La comuna que presenta los mayores PPA y PPT es la comuna de Traiguén, de la Región de La Araucanía, con 1,86 (intervalo de confianza (IC) 0,40-2,74) y 1,60 (IC 0,33-2,54), respectivamente. Por otra parte, las comunas con menores PPA y PPT son Conchalí y Cerro Navia, ambas de la Región Metropolitana de Santiago, con 0,30 (IC 0,29-0,32) y 0,25 (IC 0,25-0,31), respectivamente.

Es así como se observa que la comuna con mayor desigualdad y mayor polarización social, entre las 78 comunas analizadas, es la comuna de Traiguén, de la Región de La Araucanía, y en contraposición Cerro Navia, de la Región Metropolitana de Santiago, es la comuna con menor desigualdad y menor polarización social.

Las comunas de la Región de Coquimbo presentan un coeficiente de Gini con ingreso per cápita autónomo de entre 0,42 y 0,51 (M 0,47; DE 0,03) y un Gini con ingreso per cápita total de entre 0,35 y 0,42 (M 0,39; DE 0,02). La comuna que presenta el mayor GPA es Illapel, con 0,51 (IC 0,43-0,56), y la que presenta el mayor GPT es La Serena, con 0,42 (IC 0,40-0,43). La comuna que exhibe el menor GPA es Ovalle, con 0,42 (IC 0,39-0,42), y también la que exhibe el menor GPT, con 0,35 (IC 0,33-0,37).

El índice de polarización con ingreso per cápita autónomo se encuentra entre 0,33 y 0,43 ( $M$ 0,38; $D E$ 0,03) y el de polarización con ingreso per cápita total entre 0,28 y 0,34 ( 0 0,32; $D E$ 0,02). La comuna con mayor PPA es Coquimbo y la que presenta mayor PPT es Vicuña, en tanto que la que presenta los menores PPA y PPT es Ovalle (véase el cuadro 5).

Las comunas de la Región de Valparaíso presentan un coeficiente de Gini con ingreso per cápita autónomo de entre 0,42 y 0,53 (M 0,46; $D E$ 0,03) y un Gini con ingreso per cápita total de entre 0,34 y 0,48 (M 0,39; $D E$ 0,03). La comuna con mayores GPA y GPT es Viña del Mar, con 0,53 (IC 0,50-0,56) y 0,48 (IC 0,45-0,51), respectivamente. La comuna con menores GPA y GPT es San Antonio, con 0,42 (IC 0,38-0,44) y 0,35 (IC 0,32-0,37), respectivamente. 
El índice de polarización con ingreso per cápita autónomo se encuentra entre 0,34 y 0,85 $(M 0,43 ; D E 0,16)$ y el de polarización con ingreso per cápita total entre 0,28 y $0,75(M 0,36 ; D E 0,12)$. La comuna que presenta los mayores PPA y PPT es Viña del Mar, y las que presentan los menores PPA y PPT son El Quisco y La Ligua, respectivamente (véase el cuadro 6).

\section{Cuadro 5}

Región de Coquimbo: coeficientes de Gini e índices de polarización con intervalos de confianza

\begin{tabular}{|c|c|c|c|c|c|}
\hline Comunas & Población total & $\begin{array}{c}\text { GPA } \\
\text { (IC } 95 \%) \\
\end{array}$ & $\begin{array}{c}\text { GPT } \\
\text { (IC 95\%) } \\
\end{array}$ & $\begin{array}{c}\text { PPA } \\
\text { (IC 95\%) }\end{array}$ & $\begin{array}{c}\text { PPT } \\
\text { (IC 95\%) }\end{array}$ \\
\hline Coquimbo & 257931 & $\begin{array}{c}0,46 \\
(0,43-0,49)\end{array}$ & $\begin{array}{c}0,39 \\
(0,36-0,42)\end{array}$ & $\begin{array}{c}0,43 \\
(0,39-0,50)\end{array}$ & $\begin{array}{c}0,34 \\
(0,31-0,41)\end{array}$ \\
\hline Illapel & 32964 & $\begin{array}{c}0,51 \\
(0,43-0,57) \\
\end{array}$ & $\begin{array}{c}0,40 \\
(0,33-0,46)\end{array}$ & $\begin{array}{c}0,41 \\
(0,38-0,56)\end{array}$ & $\begin{array}{c}0,33 \\
(0,30-0,43)\end{array}$ \\
\hline La Serena & 237433 & $\begin{array}{c}0,48 \\
(0,45-0,50)\end{array}$ & $\begin{array}{c}0,42 \\
(0,39-0,44)\end{array}$ & $\begin{array}{c}0,38 \\
(0,37-0,44)\end{array}$ & $\begin{array}{c}0,33 \\
(0,33-0,38)\end{array}$ \\
\hline Ovalle & 127072 & $\begin{array}{c}0,42 \\
(0,38-0,44)\end{array}$ & $\begin{array}{c}0,35 \\
(0,32-0,37)\end{array}$ & $\begin{array}{c}0,33 \\
(0,33-0,39)\end{array}$ & $\begin{array}{c}0,28 \\
(0,28-0,32)\end{array}$ \\
\hline Vicuña & 27069 & $\begin{array}{c}0,49 \\
(0,42-0,53)\end{array}$ & $\begin{array}{c}0,41 \\
(0,36-0,45)\end{array}$ & $\begin{array}{c}0,39 \\
(0,37-0,49)\end{array}$ & $\begin{array}{c}0,34 \\
(0,33-0,42)\end{array}$ \\
\hline
\end{tabular}

Fuente: Elaboración propia, sobre la base de datos de Ministerio de Desarrollo Social y Familia, "Encuesta CASEN 2015", Observatorio Social, Santiago [en línea] http://observatorio.ministeriodesarrollosocial.gob.cl/casen-multidimensional/ casen/casen_2015.php.

Nota: GPA: Gini con ingreso per cápita autónomo; GPT: Gini con ingreso per cápita total; PPA: Polarización con ingreso per cápita autónomo; PPT: Polarización con ingreso per cápita total.

\section{Cuadro 6}

Región de Valparaíso: coeficientes de Gini e índices de polarización con intervalos de confianza

\begin{tabular}{|c|c|c|c|c|c|}
\hline Comunas & Población total & $\begin{array}{c}\text { GPA } \\
\text { (IC 95\%) }\end{array}$ & $\begin{array}{c}\text { GPT } \\
(I C 95 \%)\end{array}$ & $\begin{array}{c}\text { PPA } \\
\text { (IC 95\%) }\end{array}$ & $\begin{array}{c}\text { PPT } \\
\text { (IC 95\%) }\end{array}$ \\
\hline Concón & 55805 & $\begin{array}{c}0,49 \\
(0,44-0,53)\end{array}$ & $\begin{array}{c}0,43 \\
(0,38-0,46)\end{array}$ & $\begin{array}{c}0,38 \\
(0,38-0,52)\end{array}$ & $\begin{array}{c}0,34 \\
(0,34-0,44)\end{array}$ \\
\hline El Quisco & 14479 & $\begin{array}{c}0,43 \\
(0,39-0,47)\end{array}$ & $\begin{array}{c}0,35 \\
(0,31-0,38)\end{array}$ & $\begin{array}{c}0,34 \\
(0,32-0,40)\end{array}$ & $\begin{array}{c}0,28 \\
(0,27-0,33)\end{array}$ \\
\hline La Calera & 56067 & $\begin{array}{c}0,46 \\
(0,38-0,51)\end{array}$ & $\begin{array}{c}0,39 \\
(0,33-0,44)\end{array}$ & $\begin{array}{c}0,39 \\
(0,37-0,52)\end{array}$ & $\begin{array}{c}0,35 \\
(0,32-0,46)\end{array}$ \\
\hline La Ligua & 33803 & $\begin{array}{c}0,43 \\
(0,38-0,47)\end{array}$ & $\begin{array}{c}0,34 \\
(0,30-0,37)\end{array}$ & $\begin{array}{c}0,34 \\
(0,32-0,39)\end{array}$ & $\begin{array}{c}0,28 \\
(0,26-0,32)\end{array}$ \\
\hline Limache & 46870 & $\begin{array}{c}0,44 \\
(0,40-0,47)\end{array}$ & $\begin{array}{c}0,38 \\
(0,35-0,41)\end{array}$ & $\begin{array}{c}0,37 \\
(0,34-0,49)\end{array}$ & $\begin{array}{c}0,34 \\
(0,32-0,55)\end{array}$ \\
\hline Los Andes & 69609 & $\begin{array}{c}0,44 \\
(0,39-0,47)\end{array}$ & $\begin{array}{c}0,38 \\
(0,34-0,42)\end{array}$ & $\begin{array}{c}0,36 \\
(0,35-0,46)\end{array}$ & $\begin{array}{c}0,32 \\
(0,32-0,42)\end{array}$ \\
\hline Quillota & 99063 & $\begin{array}{c}0,48 \\
(0,41-0,53)\end{array}$ & $\begin{array}{c}0,41 \\
(0,35-0,45)\end{array}$ & $\begin{array}{c}0,40 \\
(0,38-0,54)\end{array}$ & $\begin{array}{c}0,35 \\
(0,34-0,47)\end{array}$ \\
\hline Quilpué & 181831 & $\begin{array}{c}0,47 \\
(0,42-0,51)\end{array}$ & $\begin{array}{c}0,41 \\
(0,36-0,44)\end{array}$ & $\begin{array}{c}0,42 \\
(0,35-0,51)\end{array}$ & $\begin{array}{c}0,33 \\
(0,31-0,21)\end{array}$ \\
\hline San Antonio & 98299 & $\begin{array}{c}0,42 \\
(0,38-0,45)\end{array}$ & $\begin{array}{c}0,35 \\
(0,31-0,38)\end{array}$ & $\begin{array}{c}0,34 \\
(0,33-0,43)\end{array}$ & $\begin{array}{c}0,28 \\
(0,28-0,34)\end{array}$ \\
\hline San Felipe & 76103 & $\begin{array}{c}0,47 \\
(0,43-0,49)\end{array}$ & $\begin{array}{c}0,40 \\
(0,37-0,43)\end{array}$ & $\begin{array}{c}0,35 \\
(0,35-0,41)\end{array}$ & $\begin{array}{c}0,32 \\
(0,31-0,36)\end{array}$ \\
\hline Valparaíso & 295916 & $\begin{array}{c}0,48 \\
(0,44-0,52)\end{array}$ & $\begin{array}{c}0,43 \\
(0,39-0,47)\end{array}$ & $\begin{array}{c}0,72 \\
(0,37-0,82)\end{array}$ & $\begin{array}{c}0,41 \\
(0,33-0,69)\end{array}$ \\
\hline Villa Alemana & 155527 & $\begin{array}{c}0,45 \\
(0,41-0,47)\end{array}$ & $\begin{array}{c}0,40 \\
(0,37-0,43)\end{array}$ & $\begin{array}{c}0,37 \\
(0,37-0,45)\end{array}$ & $\begin{array}{c}0,33 \\
(0,32-0,39)\end{array}$ \\
\hline Viña del Mar & 330898 & $\begin{array}{c}0,53 \\
(0,49-0,57)\end{array}$ & $\begin{array}{c}0,48 \\
(0,44-0,51)\end{array}$ & $\begin{array}{c}0,85 \\
(0,48-0,95)\end{array}$ & $\begin{array}{c}0,75 \\
(0,42-0,83)\end{array}$ \\
\hline
\end{tabular}

Fuente: Elaboración propia, sobre la base de datos de Ministerio de Desarrollo Social y Familia, "Encuesta CASEN 2015", Observatorio Social, Santiago [en línea] http://observatorio.ministeriodesarrollosocial.gob.cl/casen-multidimensional/ casen/casen_2015.php.

Nota: GPA: Gini con ingreso per cápita autónomo; GPT: Gini con ingreso per cápita total; PPA: Polarización con ingreso per cápita autónomo; PPT: Polarización con ingreso per cápita total. 
La Región del Biobío presenta comunas con coeficientes de Gini con ingreso per cápita autónomo de entre 0,40 y 0,56 ( $M 0,47 ; D E 0,053)$ y Gini con ingreso per cápita total de entre 0,32 y 0,50 ( $M$ 0,38; $D E$ 0,05). La comuna que exhibe el mayor GPA es San Pedro de la Paz, con 0,56 (IC 0,52-0,58), que también presenta el mayor GPT, de 0,50 (IC 0,47-0,53). Por otra parte, la comuna que presenta menores GPA y GPT es Hualpén, con 0,40 (IC 0,35-0,43) y 0,32 (IC 0,28-0,34), respectivamente.

El índice de polarización con ingreso per cápita autónomo se encuentra entre 0,34 y 0,92 ( $M$ 0,42; $D E 0,13)$ y el de polarización con ingreso per cápita total entre 0,27 y $0,78(M 0,33 ; D E 0,11)$. La comuna que presenta el mayor PPA y el mayor PPT es Chillán, con 0,92 (IC 0,46-1,11) y 0,78 (IC 0,36-0,95), respectivamente. La que exhibe el menor PPA y el menor PPT es Curanilahue, con 0,34 (IC 0,33-0,40) y 0,27 (IC 0,26-0,032), respectivamente (véase el cuadro 7).

\section{Cuadro 7}

Región del Biobío: coeficientes de Gini e índices de polarización con intervalos de confianza

\begin{tabular}{|c|c|c|c|c|c|}
\hline Comunas & Población total & $\begin{array}{c}\text { GPA } \\
\text { (IC } 95 \%)\end{array}$ & $\begin{array}{c}\text { GPT } \\
\text { (IC } 95 \%)\end{array}$ & $\begin{array}{c}\text { PPA } \\
(I C 95 \%)\end{array}$ & $\begin{array}{c}\text { PPT } \\
\text { (IC 95\%) }\end{array}$ \\
\hline Arauco & 38521 & $\begin{array}{c}0,46 \\
(0,41-0,50)\end{array}$ & $\begin{array}{c}0,33 \\
(0,29-0,36)\end{array}$ & $\begin{array}{c}0,41 \\
(0,40-0,53)\end{array}$ & $\begin{array}{c}0,30 \\
(0,29-0,37)\end{array}$ \\
\hline Cañete & 34214 & $\begin{array}{c}0,51 \\
(0,47-0,53)\end{array}$ & $\begin{array}{c}0,41 \\
(0,37-0,43)\end{array}$ & $\begin{array}{c}0,38 \\
(0,37-0,43)\end{array}$ & $\begin{array}{c}0,31 \\
(0,30-0,35)\end{array}$ \\
\hline Chiguayante & 104382 & $\begin{array}{c}0,44 \\
(0,40-0,46)\end{array}$ & $\begin{array}{c}0,36 \\
(0,32-0,38)\end{array}$ & $\begin{array}{c}0,35 \\
(0,35-0,42)\end{array}$ & $\begin{array}{c}0,29 \\
(0,29-0,35)\end{array}$ \\
\hline Chillán & 182622 & $\begin{array}{c}0,55 \\
(0,48-0,61)\end{array}$ & $\begin{array}{c}0,46 \\
(0,39-0,53)\end{array}$ & $\begin{array}{c}0,92 \\
(0,46-1,11)\end{array}$ & $\begin{array}{c}0,78 \\
(0,36-0,95)\end{array}$ \\
\hline Chillán Viejo & 36553 & $\begin{array}{c}0,45 \\
(0,36-0,51)\end{array}$ & $\begin{array}{c}0,37 \\
(0,28-0,43)\end{array}$ & $\begin{array}{c}0,41 \\
(0,37-0,58)\end{array}$ & $\begin{array}{c}0,32 \\
(0,30-0,50)\end{array}$ \\
\hline Concepción & 228779 & $\begin{array}{c}0,51 \\
(0,47-0,53)\end{array}$ & $\begin{array}{c}0,44 \\
(0,40-0,46)\end{array}$ & $\begin{array}{c}0,47 \\
(0,46-0,57)\end{array}$ & $\begin{array}{c}0,39 \\
(0,37-0,49)\end{array}$ \\
\hline Coronel & 120729 & $\begin{array}{c}0,41 \\
(0,37-0,43)\end{array}$ & $\begin{array}{c}0,33 \\
(0,30-0,35)\end{array}$ & $\begin{array}{c}0,34 \\
(0,33-0,42)\end{array}$ & $\begin{array}{c}0,27 \\
(0,27-0,32)\end{array}$ \\
\hline Curanilahue & 35157 & $\begin{array}{c}0,43 \\
(0,39-0,46)\end{array}$ & $\begin{array}{c}0,34 \\
(0,30-0,37)\end{array}$ & $\begin{array}{c}0,34 \\
(0,33-0,40)\end{array}$ & $\begin{array}{c}0,27 \\
(0,26-0,32)\end{array}$ \\
\hline Hualpén & 114833 & $\begin{array}{c}0,40 \\
(0,35-0,43)\end{array}$ & $\begin{array}{c}0,32 \\
(0,28-0,34)\end{array}$ & $\begin{array}{c}0,35 \\
(0,35-0,44)\end{array}$ & $\begin{array}{c}0,28 \\
(0,28-0,35)\end{array}$ \\
\hline Laja & 24079 & $\begin{array}{c}0,56 \\
(0,46-0,64)\end{array}$ & $\begin{array}{c}0,43 \\
(0,32-0,52)\end{array}$ & $\begin{array}{c}0,53 \\
(0,42-0,73)\end{array}$ & $\begin{array}{c}0,35 \\
(0,29-0,50)\end{array}$ \\
\hline Lebu & 26791 & $\begin{array}{c}0,55 \\
(0,45-0,62)\end{array}$ & $\begin{array}{c}0,47 \\
(0,38-0,53)\end{array}$ & $\begin{array}{c}0,46 \\
(0,41-0,65)\end{array}$ & $\begin{array}{c}0,38 \\
(0,35-0,52)\end{array}$ \\
\hline Los Ángeles & 202214 & $\begin{array}{c}0,48 \\
(0,46-0,50)\end{array}$ & $\begin{array}{c}0,42 \\
(0,39-0,43)\end{array}$ & $\begin{array}{c}0,40 \\
(0,39-0,45)\end{array}$ & $\begin{array}{c}0,32 \\
(0,31-0,35)\end{array}$ \\
\hline Lota & 46241 & $\begin{array}{c}0,42 \\
(0,37-0,47)\end{array}$ & $\begin{array}{c}0,34 \\
(0,29-0,39)\end{array}$ & $\begin{array}{c}0,34 \\
(0,33-0,43)\end{array}$ & $\begin{array}{c}0,27 \\
(0,26-0,34)\end{array}$ \\
\hline Mulchén & 30354 & $\begin{array}{c}0,47 \\
(0,39-0,53)\end{array}$ & $\begin{array}{c}0,35 \\
(0,28-0,40)\end{array}$ & $\begin{array}{c}0,38 \\
(0,36-0,51)\end{array}$ & $\begin{array}{c}0,29 \\
(0,27-0,40)\end{array}$ \\
\hline Nacimiento & 28699 & $\begin{array}{c}0,46 \\
(0,40-0,51)\end{array}$ & $\begin{array}{c}0,37 \\
(0,32-0,41)\end{array}$ & $\begin{array}{c}0,37 \\
(0,36-0,45)\end{array}$ & $\begin{array}{c}0,30 \\
(0,29-0,37)\end{array}$ \\
\hline Penco & 52695 & $\begin{array}{c}0,42 \\
(0,36-0,48)\end{array}$ & $\begin{array}{c}0,34 \\
(0,29-0,39)\end{array}$ & $\begin{array}{c}0,35 \\
(0,34-0,48)\end{array}$ & $\begin{array}{c}0,29 \\
(0,29-0,41)\end{array}$ \\
\hline San Pedro de la Paz & 153562 & $\begin{array}{c}0,56 \\
(0,51-0,59)\end{array}$ & $\begin{array}{c}0,50 \\
(0,46-0,53)\end{array}$ & $\begin{array}{c}0,45 \\
(0,43-0,55)\end{array}$ & $\begin{array}{c}0,39 \\
(0,38-0,47)\end{array}$ \\
\hline Talcahuano & 179670 & $\begin{array}{c}0,44 \\
(0,41-0,47)\end{array}$ & $\begin{array}{c}0,38 \\
(0,34-0,40)\end{array}$ & $\begin{array}{c}0,37 \\
(0,36-0,45)\end{array}$ & $\begin{array}{c}0,30 \\
(0,30-0,38)\end{array}$ \\
\hline Tomé & 55760 & $\begin{array}{c}0,44 \\
(0,37-0,49)\end{array}$ & $\begin{array}{c}0,36 \\
(0,30-0,41)\end{array}$ & $\begin{array}{c}0,37 \\
(0,35-0,50)\end{array}$ & $\begin{array}{c}0,31 \\
(0,30-0,41)\end{array}$ \\
\hline
\end{tabular}

Fuente: Elaboración propia, sobre la base de datos de Ministerio de Desarrollo Social y Familia, "Encuesta CASEN 2015", Observatorio Social, Santiago [en línea] http://observatorio.ministeriodesarrollosocial.gob.cl/casen-multidimensional/ casen/casen_2015.php.

Nota: GPA: Gini con ingreso per cápita autónomo; GPT: Gini con ingreso per cápita total; PPA: Polarización con ingreso per cápita autónomo; PPT: Polarización con ingreso per cápita total.

Las comunas de la Región de La Araucanía presentan coeficientes de Gini con ingreso per cápita autónomo de entre 0,45 y 0,81 (M 0,52; DE 0,087) y de Gini con ingreso per cápita total de entre 0,35 y 0,74 (M 0,41; DE 0,095). La comuna que presenta el mayor GPA es Traiguén, con 0,81 
(IC 0,46-0,90), y exhibe también el mayor GPT, de 0,74 (IC 0,37-0,85). Las comunas que presentan el menor GPA son Freire, con 0,45 (IC 0,39-0,49), y Padre Las Casas, también con 0,45 (IC 0,41-0,48), en tanto que el menor GPT corresponde a Freire, con 0,33 (IC 0,28-0,36).

El índice de polarización con ingreso per cápita autónomo se encuentra entre 0,36 y 1,86 ( $M$ 0,49; $D E$ 0,37) y el de polarización con ingreso per cápita total entre 0,28 y 1,60 ( $M$ 0,40; $D E$ 0,33). La comuna que presenta el mayor PPA y el mayor PPT es Traiguén, con 1,86 (IC 0,40-2,74) y 1,60 (IC 0,33-2,54), respectivamente. La comuna que exhibe el menor PPA es Padre Las Casas, con 0,36 (IC 0,35-0,43), y la que presenta el menor PPT es Freire, con 0,28 (IC 0,26-0,34) (véase el cuadro 8).

\section{Cuadro 8}

Región de La Araucanía: coeficientes de Gini e índices de polarización con intervalos de confianza

\begin{tabular}{|c|c|c|c|c|c|}
\hline Comunas & Población total & $\begin{array}{c}\text { GPA } \\
\text { (IC 95\%) }\end{array}$ & $\begin{array}{c}\text { GPT } \\
\text { (IC 95\%) }\end{array}$ & $\begin{array}{c}\text { PPA } \\
\text { (IC 95\%) }\end{array}$ & $\begin{array}{c}\text { PPT } \\
\text { (IC 95\%) }\end{array}$ \\
\hline Angol & 56563 & $\begin{array}{c}0,48 \\
(0,43-0,51)\end{array}$ & $\begin{array}{c}0,38 \\
(0,34-0,41)\end{array}$ & $\begin{array}{c}0,37 \\
(0,36-0,46)\end{array}$ & $\begin{array}{c}0,30 \\
(0,29-0,34)\end{array}$ \\
\hline Carahue & 26276 & $\begin{array}{c}0,58 \\
(0,45-0,69)\end{array}$ & $\begin{array}{c}0,44 \\
(0,31-0,56)\end{array}$ & $\begin{array}{c}0,48 \\
(0,38-0,74)\end{array}$ & $\begin{array}{c}0,35 \\
(0,29-0,58)\end{array}$ \\
\hline Collipulli & 24875 & $\begin{array}{c}0,52 \\
(0,46-0,56)\end{array}$ & $\begin{array}{c}0,42 \\
(0,36-0,46)\end{array}$ & $\begin{array}{c}0,39 \\
(0,36-0,46)\end{array}$ & $\begin{array}{c}0,32 \\
(0,30-0,38)\end{array}$ \\
\hline Cunco & 18724 & $\begin{array}{c}0,50 \\
(0,42-0,57)\end{array}$ & $\begin{array}{c}0,35 \\
(0,28-0,41)\end{array}$ & $\begin{array}{c}0,39 \\
(0,36-0,53)\end{array}$ & $\begin{array}{c}0,28 \\
(0,26-0,36)\end{array}$ \\
\hline Curacautín & 16907 & $\begin{array}{c}0,55 \\
(0,45-0,63)\end{array}$ & $\begin{array}{c}0,41 \\
(0,33-0,51)\end{array}$ & $\begin{array}{c}0,47 \\
(0,37-0,63)\end{array}$ & $\begin{array}{c}0,32 \\
(0,28-0,47)\end{array}$ \\
\hline Freire & 23867 & $\begin{array}{c}0,45 \\
(0,39-0,49)\end{array}$ & $\begin{array}{c}0,33 \\
(0,28-0,37)\end{array}$ & $\begin{array}{c}0,37 \\
(0,35-0,46)\end{array}$ & $\begin{array}{c}0,28 \\
(0,26-0,34)\end{array}$ \\
\hline Lautaro & 37952 & $\begin{array}{c}0,49 \\
(0,45-0,53)\end{array}$ & $\begin{array}{c}0,39 \\
(0,35-0,43)\end{array}$ & $\begin{array}{c}0,40 \\
(0,39-0,48)\end{array}$ & $\begin{array}{c}0,32 \\
(0,31-0,39)\end{array}$ \\
\hline Nueva Imperial & 33976 & $\begin{array}{c}0,47 \\
(0,42-0,51)\end{array}$ & $\begin{array}{c}0,35 \\
(0,31-0,38)\end{array}$ & $\begin{array}{c}0,38 \\
(0,37-0,46)\end{array}$ & $\begin{array}{c}0,28 \\
(0,28-0,34)\end{array}$ \\
\hline Padre Las Casas & 98459 & $\begin{array}{c}0,45 \\
(0,40-0,49)\end{array}$ & $\begin{array}{c}0,36 \\
(0,32-0,40)\end{array}$ & $\begin{array}{c}0,36 \\
(0,35-0,43)\end{array}$ & $\begin{array}{c}0,30 \\
(0,28-0,36)\end{array}$ \\
\hline Pitrufquén & 25184 & $\begin{array}{c}0,51 \\
(0,45-0,55)\end{array}$ & $\begin{array}{c}0,40 \\
(0,34-0,45)\end{array}$ & $\begin{array}{c}0,38 \\
(0,36-0,46)\end{array}$ & $\begin{array}{c}0,31 \\
(0,29-0,38)\end{array}$ \\
\hline Pucón & 29991 & $\begin{array}{c}0,48 \\
(0,43-0,52)\end{array}$ & $\begin{array}{c}0,39 \\
(0,34-0,43)\end{array}$ & $\begin{array}{c}0,39 \\
(0,38-0,51)\end{array}$ & $\begin{array}{c}0,33 \\
(0,32-0,40)\end{array}$ \\
\hline Temuco & 298974 & $\begin{array}{c}0,50 \\
(0,46-0,53)\end{array}$ & $\begin{array}{c}0,43 \\
(0,39-0,46)\end{array}$ & $\begin{array}{c}0,46 \\
(0,43-0,58)\end{array}$ & $\begin{array}{c}0,39 \\
(0,36-0,49)\end{array}$ \\
\hline Traiguén & 19473 & $\begin{array}{c}0,81 \\
(0,48-0,90)\end{array}$ & $\begin{array}{c}0,74 \\
(0,39-0,86)\end{array}$ & $\begin{array}{c}1,86 \\
(0,40-2,74)\end{array}$ & $\begin{array}{c}1,60 \\
(0,33-2,54)\end{array}$ \\
\hline Victoria & 34674 & $\begin{array}{c}0,52 \\
(0,47-0,55)\end{array}$ & $\begin{array}{c}0,44 \\
(0,39-0,47)\end{array}$ & $\begin{array}{c}0,37 \\
(0,36-0,43)\end{array}$ & $\begin{array}{c}0,33 \\
(0,31-0,38)\end{array}$ \\
\hline Villarrica & 57753 & $\begin{array}{c}0,51 \\
(0,46-0,54)\end{array}$ & $\begin{array}{c}0,40 \\
(0,35-0,44)\end{array}$ & $\begin{array}{c}0,38 \\
(0,37-0,48)\end{array}$ & $\begin{array}{c}0,32 \\
(0,31-0,40)\end{array}$ \\
\hline
\end{tabular}

Fuente: Elaboración propia, sobre la base de datos de Ministerio de Desarrollo Social y Familia, "Encuesta CASEN 2015", Observatorio Social, Santiago [en línea] http://observatorio.ministeriodesarrollosocial.gob.cl/casen-multidimensional/ casen/casen_2015.php.

Nota: GPA: Gini con ingreso per cápita autónomo; GPT: Gini con ingreso per cápita total; PPA: Polarización con ingreso per cápita autónomo; PPT: Polarización con ingreso per cápita total.

Las comunas de la Región Metropolitana de Santiago presentan coeficientes de Gini con ingreso per cápita autónomo de entre 0,36 y 0,60 (M 0,42; DE 0,088) y de Gini con ingreso per cápita total de entre 0,29 y 0,53 ( $M$ 0,37; DE 0,061). La comuna que exhibe los mayores GPA y GPT es Talagante, con $0,60(I C 0,44-0,69)$ y 0,53 (IC 0,39 - 0,62), respectivamente. Por otra parte, Cerro Navia es la comuna que presenta un menor GPA, con 0,36 (IC 0,32-0,39), y un menor GPT, con 0,29 (IC 0,26-0,31).

El índice de polarización con ingreso per cápita autónomo se encuentra entre 0,34 y 0,85 ( $M$ 0,43; $D E 0,16)$ y el de polarización con ingreso per cápita total entre 0,28 y 0,75 ( $M 0,36 ; D E 0,12)$. La comuna que presenta el mayor PPA y el mayor PPT es Talagante, con 0,87 (IC 0,47-1,15) y 0,53 (IC 0,41-0,98), respectivamente. Las comunas que exhiben los valores de polarización más bajos son Conchalí, con un PPA de 0,30 (IC 0,29-0,32) y Cerro Navia, con un PPT de 0,25 (IC 0,25-0,31) (véase el cuadro 9). 


\section{Cuadro 9}

Región Metropolitana de Santiago: coeficientes de Gini e índices de polarización con intervalos de confianza

\begin{tabular}{|c|c|c|c|c|c|}
\hline Comunas & Población total & $\begin{array}{c}\text { GPA } \\
\text { (IC } 95 \%)\end{array}$ & $\begin{array}{c}\text { GPT } \\
\text { (IC 95\%) }\end{array}$ & $\begin{array}{c}\text { PPA } \\
\text { (IC 95\%) }\end{array}$ & $\begin{array}{c}\text { PPT } \\
\text { (IC 95\%) }\end{array}$ \\
\hline Cerro Navia & 158670 & $\begin{array}{c}0,36 \\
(0,32-0,39)\end{array}$ & $\begin{array}{c}0,29 \\
(0,25-0,31)\end{array}$ & $\begin{array}{c}0,31 \\
(0,30-0,37)\end{array}$ & $\begin{array}{c}0,25 \\
(0,25-0,31)\end{array}$ \\
\hline Colina & 140475 & $\begin{array}{c}0,38 \\
(0,33-0,42)\end{array}$ & $\begin{array}{c}0,34 \\
(0,29-0,38)\end{array}$ & $\begin{array}{c}0,35 \\
(0,34-0,48)\end{array}$ & $\begin{array}{c}0,29 \\
(0,30-0,41)\end{array}$ \\
\hline Conchalí & 140988 & $\begin{array}{c}0,40 \\
(0,37-0,41)\end{array}$ & $\begin{array}{c}0,33 \\
(0,31-0,34)\end{array}$ & $\begin{array}{c}0,30 \\
(0,29-0,32)\end{array}$ & $\begin{array}{c}0,26 \\
(0,25-0,27)\end{array}$ \\
\hline El Bosque & 196166 & $\begin{array}{c}0,41 \\
(0,38-0,43)\end{array}$ & $\begin{array}{c}0,34 \\
(0,32-0,36)\end{array}$ & $\begin{array}{c}0,31 \\
(0,31-0,36)\end{array}$ & $\begin{array}{c}0,27 \\
(0,26-0,29)\end{array}$ \\
\hline Estación Central & 148400 & $\begin{array}{c}0,37 \\
(0,32-0,40)\end{array}$ & $\begin{array}{c}0,32 \\
(0,28-0,34)\end{array}$ & $\begin{array}{c}0,32 \\
(0,33-0,43)\end{array}$ & $\begin{array}{c}0,29 \\
(0,29-0,36)\end{array}$ \\
\hline La Florida & 390403 & $\begin{array}{c}0,43 \\
(0,39-0,46)\end{array}$ & $\begin{array}{c}0,37 \\
(0,33-0,40)\end{array}$ & $\begin{array}{c}0,40 \\
(0,40-0,52)\end{array}$ & $\begin{array}{c}0,34 \\
(0,35-0,45)\end{array}$ \\
\hline La Granja & 144260 & $\begin{array}{c}0,37 \\
(0,32-0,40)\end{array}$ & $\begin{array}{c}0,32 \\
(0,28-0,35)\end{array}$ & $\begin{array}{c}0,32 \\
(0,32-0,40)\end{array}$ & $\begin{array}{c}0,28 \\
(0,27-0,36)\end{array}$ \\
\hline La Pintana & 217034 & $\begin{array}{c}0,37 \\
(0,33-0,39)\end{array}$ & $\begin{array}{c}0,31 \\
(0,28-0,34)\end{array}$ & $\begin{array}{c}0,32 \\
(0,32-0,39)\end{array}$ & $\begin{array}{c}0,27 \\
(0,27-0,33)\end{array}$ \\
\hline Las Condes & 290869 & $\begin{array}{c}0,45 \\
(0,42-0,47)\end{array}$ & $\begin{array}{c}0,40 \\
(0,37-0,42)\end{array}$ & $\begin{array}{c}0,34 \\
(034-0,40)\end{array}$ & $\begin{array}{c}0,32 \\
(0,32-0,37)\end{array}$ \\
\hline Macul & 125855 & $\begin{array}{c}0,53 \\
(0,40-0,62)\end{array}$ & $\begin{array}{c}0,50 \\
(0,34-0,62)\end{array}$ & $\begin{array}{c}0,52 \\
(0,39-0,79)\end{array}$ & $\begin{array}{c}0,52 \\
(0,34-0,85)\end{array}$ \\
\hline Maipú & 571632 & $\begin{array}{c}0,40 \\
(0,37-0,42)\end{array}$ & $\begin{array}{c}0,35 \\
(0,33-0,37)\end{array}$ & $\begin{array}{c}0,40 \\
(0,41-0,51)\end{array}$ & $\begin{array}{c}0,34 \\
(0,35-0,43)\end{array}$ \\
\hline Melipilla & 123669 & $\begin{array}{c}0,45 \\
(0,39-0,49)\end{array}$ & $\begin{array}{c}0,37 \\
(0,32-0,41)\end{array}$ & $\begin{array}{c}0,43 \\
(0,41-0,56)\end{array}$ & $\begin{array}{c}0,32 \\
(0,32-0,44)\end{array}$ \\
\hline Ñuñoa & 242287 & $\begin{array}{c}0,51 \\
(0,47-0,56)\end{array}$ & $\begin{array}{c}0,47 \\
(0,42-0,51)\end{array}$ & $\begin{array}{c}0,41 \\
(0,38-0,53)\end{array}$ & $\begin{array}{c}0,36 \\
(0,34-0,48)\end{array}$ \\
\hline Pedro Aguirre Cerda & 122600 & $\begin{array}{c}0,40 \\
(0,36-0,43)\end{array}$ & $\begin{array}{c}0,33 \\
(0,29-0,35)\end{array}$ & $\begin{array}{c}0,32 \\
(0,31-0,37)\end{array}$ & $\begin{array}{c}0,28 \\
(0,27-0,32)\end{array}$ \\
\hline Peñalolén & 246871 & $\begin{array}{c}0,49 \\
(0,45-0,53)\end{array}$ & $\begin{array}{c}0,44 \\
(0,39-0,47)\end{array}$ & $\begin{array}{c}0,51 \\
(0,46-0,78)\end{array}$ & $\begin{array}{c}0,44 \\
(0,41-0,65)\end{array}$ \\
\hline Providencia & 155166 & $\begin{array}{c}0,45 \\
(0,42-0,48)\end{array}$ & $\begin{array}{c}0,41 \\
(0,38-0,43)\end{array}$ & $\begin{array}{c}0,34 \\
(0,34-0,41)\end{array}$ & $\begin{array}{c}0,32 \\
(0,31-0,38)\end{array}$ \\
\hline Pudahuel & 244395 & $\begin{array}{c}0,40 \\
(0,36-0,42)\end{array}$ & $\begin{array}{c}0,34 \\
(0,31-0,36)\end{array}$ & $\begin{array}{c}0,40 \\
(0,36-0,51)\end{array}$ & $\begin{array}{c}0,32 \\
(0,31-0,43)\end{array}$ \\
\hline Puente Alto & 647428 & $\begin{array}{c}0,41 \\
(0,39-0,42)\end{array}$ & $\begin{array}{c}0,36 \\
(0,34-0,37)\end{array}$ & $\begin{array}{c}0,36 \\
(0,33-0,44)\end{array}$ & $\begin{array}{c}0,33 \\
(0,30-0,39)\end{array}$ \\
\hline Quilicura & 248306 & $\begin{array}{c}0,37 \\
(0,33-0,41)\end{array}$ & $\begin{array}{c}0,34 \\
(0,30-0,38)\end{array}$ & $\begin{array}{c}0,42 \\
(0,34-0,60)\end{array}$ & $\begin{array}{c}0,35 \\
(0,30-0,54)\end{array}$ \\
\hline Quinta Normal & 117930 & $\begin{array}{c}0,39 \\
(0,34-0,42)\end{array}$ & $\begin{array}{c}0,35 \\
(0,29-0,40)\end{array}$ & $\begin{array}{c}0,35 \\
(0,34-0,46)\end{array}$ & $\begin{array}{c}0,32 \\
(0,29-0,46)\end{array}$ \\
\hline Recoleta & 172820 & $\begin{array}{c}0,44 \\
(0,41-0,47)\end{array}$ & $\begin{array}{c}0,39 \\
(0,35-0,41)\end{array}$ & $\begin{array}{c}0,37 \\
(0,36-0,45)\end{array}$ & $\begin{array}{c}0,32 \\
(0,31-0,38)\end{array}$ \\
\hline Renca & 155465 & $\begin{array}{c}0,38 \\
(0,34-0,40)\end{array}$ & $\begin{array}{c}0,31 \\
(0,28-0,33)\end{array}$ & $\begin{array}{c}0,32 \\
(0,32-0,38)\end{array}$ & $\begin{array}{c}0,27 \\
(0,28-0,32)\end{array}$ \\
\hline San Bernardo & 312169 & $\begin{array}{c}0,45 \\
(0,39-0,49)\end{array}$ & $\begin{array}{c}0,40 \\
(0,35-0,44)\end{array}$ & $\begin{array}{c}0,43 \\
(0,41-0,57)\end{array}$ & $\begin{array}{c}0,36 \\
(0,35-0,49)\end{array}$ \\
\hline San Miguel & 122562 & $\begin{array}{c}0,50 \\
(0,47-0,53)\end{array}$ & $\begin{array}{c}0,45 \\
(0,41-0,47)\end{array}$ & $\begin{array}{c}0,39 \\
(0,39-0,45)\end{array}$ & $\begin{array}{c}0,36 \\
(0,35-0,41)\end{array}$ \\
\hline Santiago & 430114 & $\begin{array}{c}0,43 \\
(0,39-0,46)\end{array}$ & $\begin{array}{c}0,42 \\
(0,38-0,44)\end{array}$ & $\begin{array}{c}0,41 \\
(0,40-0,54)\end{array}$ & $\begin{array}{c}0,39 \\
(0,39-0,52)\end{array}$ \\
\hline Talagante & 73748 & $\begin{array}{c}0,60 \\
(0,44-0,69) \\
\end{array}$ & $\begin{array}{c}0,53 \\
(0,39-0,62) \\
\end{array}$ & $\begin{array}{c}0,87 \\
(0,47-1,15) \\
\end{array}$ & $\begin{array}{c}0,53 \\
(0,41-0,98) \\
\end{array}$ \\
\hline
\end{tabular}

Fuente: Elaboración propia, sobre la base de datos de Ministerio de Desarrollo Social y Familia, "Encuesta CASEN 2015", Observatorio Social, Santiago [en línea] http://observatorio.ministeriodesarrollosocial.gob.cl/casen-multidimensional/ casen/casen_2015.php.

Nota: GPA: Gini con ingreso per cápita autónomo; GPT: Gini con ingreso per cápita total; PPA: Polarización con ingreso per cápita autónomo; PPT: Polarización con ingreso per cápita total. 
En el análisis del coeficiente de Gini se encontró un "caso atípico" correspondiente a la comuna de Traiguén, que presenta coeficientes de Gini con ingreso per cápita autónomo y de Gini con ingreso per cápita total de 0,81 y 0,74, respectivamente, que se alejan de forma significativa de los valores del resto de las comunas observadas. Este caso puede ser explicado por la posibilidad de la participación de hogares del extremo más rico que proporcionaron información para la CASEN 2015 en esta comuna. Traiguén se ubica en la Región de La Araucanía y posee una población de 20.000 habitantes; un 25\% de la población se encuentra en condición de pobreza por ingreso y un 25\% en condición de pobreza multidimensional (Ministerio de Desarrollo Social, 2015). Las características propias de una comuna pequeña, ubicada en el extremo sur del país, podrían explicar el impacto en el coeficiente de Gini de la información de ingresos de unas pocas familias de ingresos altos.

La diferencia entre los coeficientes de Gini con ingreso per cápita autónomo y de Gini con ingreso per cápita total fue significativa (t $(77)=24,10 ; p<0,001)$. Esto evidenciaría el efecto sobre la distribución del ingreso de los subsidios monetarios traspasados a los hogares mediante las políticas sociales estatales, que disminuirían la desigualdad medida con el coeficiente de Gini.

Las tres comunas que presentan las mayores diferencias entre el GPA y el GPT son Cunco, Carahue y Curacautín, todas pertenecientes a la Región de La Araucanía. Las comunas con menores diferencias son Santiago, Quilicura y Macul, todas de la Región Metropolitana de Santiago (véase el cuadro 10). Esto se puede explicar considerando que las políticas sociales de transferencias monetarias están focalizadas en la población que se encuentra en situación de pobreza y pobreza extrema, y la Región de La Araucanía es aquella donde se presentan los mayores índices de pobreza del país; por lo tanto, es allí donde se concentran las transferencias monetarias del Estado a las familias.

\section{Cuadro 10}

Comunas con mayores y menores diferencias entre los coeficientes de Gini con ingreso per cápita autónomo (GPA) y de Gini con ingreso per cápita total (GPT)

\begin{tabular}{lllccc}
\hline & Región de pertenencia & Comuna & $\begin{array}{c}\text { Gini con ingreso } \\
\text { per cápita autónomo }\end{array}$ & $\begin{array}{c}\text { Gini con ingreso } \\
\text { per cápita total }\end{array}$ & Diferencia \\
\hline $\begin{array}{l}\text { Comunas con } \\
\text { mayores diferencias }\end{array}$ & La Araucanía & Cunco & 0,50 & 0,35 & 0,15 \\
\cline { 2 - 6 } & La Araucanía & Carahue & 0,58 & 0,44 & 0,14 \\
\cline { 2 - 6 } & La Araucanía & Curacautín & 0,55 & 0,41 & 0,13 \\
\hline $\begin{array}{l}\text { Comunas con } \\
\text { menores diferencias }\end{array}$ & Metropolitana & Quilicura & 0,37 & 0,34 & 0,03 \\
\cline { 2 - 6 } & Metropolitana & Macul & 0,53 & 0,50 & 0,03 \\
\cline { 2 - 6 } & Metropolitana & Santiago & 0,43 & 0,42 & 0,02 \\
\hline
\end{tabular}

Fuente: Elaboración propia.

En el análisis de la polarización social los resultados son diferentes, y no se observó una diferencia significativa en la comparación entre PPA y PPT. Finalmente se analizó la correlación existente entre desigualdad y polarización, que se presentó estadísticamente significativa.

\section{Comentarios finales}

A partir del análisis del coeficiente de Gini en las 78 comunas analizadas se observa que, aun cuando existe gran variabilidad, la desigualdad se mantiene alta en todas las comunas. El coeficiente de Gini con ingreso per cápita autónomo va de 0,36 a 0,81, y el $27 \%$ de las comunas presentan valores sobre el índice nacional $(0,49)$. En cuanto al coeficiente de Gini con ingreso per cápita total, se observan índices levemente menores, que van de 0,29 a 0,74, y el 5\% de las comunas superan el índice nacional $(0,48)$. Sin embargo, si se realiza la comparación con la media del coeficiente de Gini de los países de la $\operatorname{OCDE}(0,30)$, se observa que prácticamente el $100 \%$ de las comunas presentan valores de GPA y GPT superiores a ese promedio. 
Lo anterior viene a confirmar que, aun cuando el país ha experimentado un constante crecimiento económico y ha alcanzado cifras en indicadores sociales al nivel de países desarrollados, no se ha logrado una mejor distribución de los ingresos, lo que coincide con los hallazgos de varios estudios (Contreras, 1999; Schatan, 2005; Vergara, 2011; PNUD, 2017).

En economías reguladas por el mercado, como es el caso de Chile, la búsqueda de una mayor igualdad en la distribución de los ingresos, en particular, y de la riqueza, en general, debería ser parte de una política gubernamental que, además de incluir políticas subsidiarias focalizadas, estableciera regulaciones respecto a la acumulación de riqueza (Schatan, 2005). De esta manera, se potenciaría el bienestar de la población, disminuyendo las diferencias sociales que se producen entre los grupos como consecuencia de la estratificación social altamente polarizada. Investigaciones han demostrado que sociedades más igualitarias tienden a generar mejores condiciones para el desarrollo de la empatía entre las personas, tendencia que facilita el establecimiento de relaciones interpersonales (Jahoda, 1958) y, en general, la existencia de vínculos positivos entre personas y grupos (Ryff, 1989). Por su parte, otros investigadores han mostrado que el malestar físico y psicológico puede surgir cuando una persona se autopercibe con menor estatus social o clase al compararse con un grupo de referencia que tiene mayores privilegios (Osafo Hounkpatin y otros, 2015; Quijada y otros, 2018).

En el contexto de una economía que, por un lado, presenta indicadores de crecimiento favorables y, por otro, mantiene altos índices de desigualdad, las transferencias monetarias han cumplido un rol importante dentro de las políticas públicas. Ciertamente, estas no tienen como finalidad principal disminuir la desigualdad, sino más bien mejorar algunos indicadores de calidad de vida de la población. Con todo, los resultados de este estudio evidencian algunos efectos relevantes de analizar al comparar los coeficientes de Gini calculados con los ingresos autónomos con aquellos calculados con los ingresos totales. Es así como se observó una mayor desigualdad en todas las comunas al considerar los ingresos autónomos de un hogar. Esto reflejaría el efecto de las transferencias monetarias, en el sentido de disminuir la desigualdad; este resultado coindice con lo informado oficialmente (Ministerio de Desarrollo Social, 2017). Sin embargo, hay que tener presente que estos resultados no logran mejorar indicadores a nivel nacional, ya que Chile se mantiene como uno de los países más desiguales del mundo (OCDE, 2015). Además, el impacto de las transferencias monetarias en la redistribución sería menor que en los países de la OCDE, donde el impacto triplica el que se presenta en Chile (Martner, 2008; Aguirre Briones, 2009).

Respecto al índice de polarización los resultados son distintos, y no se observaron diferencias significativas al comparar la polarización calculada con el ingreso per cápita autónomo y la polarización calculada con el ingreso per cápita total. Como se indica más arriba, la polarización identifica distintos grupos con un alto distanciamiento entre ellos y muy similares internamente. Tal como la desigualdad, la polarización puede abordarse mediante la política de transferencias monetarias, pero para ello estas transferencias deberían ser significativamente mayores que las que se realizan en la actualidad y aplicarse de forma diferenciada según la posición en la distribución existente. De esta forma, no solo mejorarían los promedios nacionales, que en muchas ocasiones tienden a invisibilizar las realidades extremas (Vergara, 2011), que corresponden a comunas con indicadores distantes del promedio, como, por ejemplo, en el extremo más alto, las comunas de Traiguén, con coeficientes de Gini con ingreso per cápita autónomo y de Gini con ingreso per cápita total de 0,81 y 0,74, respectivamente, y de Talagante, con 0,60 y 0,53, respectivamente, y en el extremo inferior la comuna de Cerro Navia, con coeficientes de Gini con ingreso per cápita autónomo y total de 0,36 y 0,29, respectivamente.

Por lo tanto, si bien se observa que las transferencias monetarias disminuyen la desigualdad en la distribución de los ingresos, esta disminución no es suficiente para producir un cambio en la estructura de la distribución y menos aún un cambio en la conformación de conglomerados o grupos generados por el distanciamiento social. La existencia de estos conglomerados potencia el surgimiento de conflictos, ya que algunos estudios han asociado la polarización social con la reducción de la seguridad de los derechos de propiedad y contratos (Keefer y Knack, 2002) y el aumento de la probabilidad de delitos de mayor connotación social (Vergara, 2011). 
Este análisis de la desigualdad y la polarización social a nivel de comunas del país hace evidente que la alta desigualdad en la distribución de los ingresos presente a nivel nacional y regional se replica a nivel de las comunas. Esto justifica la necesidad de crear políticas de redistribuciones de ingresos focalizadas y pertinentes a este nivel territorial, ya que el impacto que generan la desigualdad y la polarización social puede estar afectando, directa e indirectamente, el bienestar percibido por los habitantes. En Chile una forma de potenciar a los territorios más desventajados ha sido, principalmente, la modificación de la división político-administrativa para crear comunas, provincias y regiones (Pressacco, 2009). Sin embargo, esto no ha sido acompañado de políticas de desarrollo descentralizadas, tendientes a una mejora de los indicadores de desigualdad e integración social (Pérez, 2011), para la reducción de los efectos negativos del distanciamiento social entre la población como consecuencia de la desigualdad existente.

Para finalizar, es importante hacer presentes las limitaciones de esta investigación. Entre ellas se encuentra la dificultad de los estudios que se realizan con información de ingresos declarados por los hogares (Schatan, 2005; Ortiz y Cummins, 2011; Atkinson, Piketty y Saez, 2011), especialmente por la dificultad de acceso a los hogares con más recursos, que no participan en estos estudios o informan ingresos menores que los reales. En Chile, la concentración de los recursos económicos se encuentra en un grupo muy reducido de familias o grupos económicos poco accesibles para estos estudios (Atria y otros, 2017). Por lo tanto, es muy probable que la desigualdad de los ingresos de los hogares sea mayor que la que se ha informado oficialmente o la que se ha estimado en estudios realizados con información proveniente de encuestas nacionales oficiales como la CASEN. Se requieren nuevos estudios transversales y longitudinales, con fuentes de información que puedan complementar las actuales para una mejor estimación de la desigualdad y la polarización, mediante la integración de información de los hogares de difícil acceso tanto por el estrato social al que pertenecen como por su ubicación en comunas que corresponden a territorios más aislados o extremos del país. Otro desafío que emerge es la realización de estudios que aborden los efectos de la polarización en diferentes contextos poblacionales y territoriales.

\section{Bibliografía}

Agostini, C. A. y P. H. Brown (2007), "Desigualdad geográfica en Chile", Revista de Análisis Económico, vol. 22, N $N^{\circ}$ 1, Santiago, Universidad Alberto Hurtado, junio.

Aguirre Briones, A. (2009), "Política social e indicadores distributivos: elementos para una caracterización de la política social en Chile", Polis: Revista Latinoamericana, № 22, Santiago, Centro de Estudios del Desarrollo Regional y Políticas Públicas (CEDER).

Alvaredo, F. y otros (coords.) (2018), World Inequality Report 2018, Laboratorio sobre la Desigualdad Global [en línea] http://wir2018.wid.world/files/download/wir2018-full-report-english.pdf.

Amarante, V. y M. Colacce (2018), "¿Más o menos desiguales? Una revisión sobre la desigualdad de los ingresos a nivel global, regional y nacional", Revista CEPAL, No 124 (LC/PUB.2018/5-P), Santiago, Comisión Económica para América Latina y el Caribe (CEPAL), abril.

Araya Rosales, A. y M. Gallardo Altamirano (2015), "El Modelo Chileno desde una ética de justicia y de igualdad de las oportunidades humanas", Polis: Revista Latinoamericana, № 40, Santiago, Centro de Estudios del Desarrollo Regional y Políticas Públicas (CEDER).

Atkinson, A. B., T. Piketty y E. Saez (2011), "Top incomes in the long run of history", Journal of Economic Literature, vol. 49, № 1, Nashville, Asociación Estadounidense de Economía, marzo.

Atria, J. y otros (2017), "Investigando a la élite económica: lecciones y desafíos a partir del caso de Chile", CUHSO, vol. 27, № 2, Temuco, Universidad Católica de Temuco, diciembre.

Banco Mundial (s/f), "Datos de libre acceso del Banco Mundial" [en línea] datos.bancomundial.org.

Campos-Arias, A. y E. Herazo (2015), "Asociación entre desigualdad y tasa de suicidio en Colombia (1994-2013)", Revista Colombiana de Psiquiatría, vol. 44, № 1, Elsevier España, enero-marzo.

Cárdenas, E. (2011), "Polarización y conflicto social", Revista de Economía Institucional, vol. 13, № 24, Bogotá, Universidad Externado de Colombia. 
Carpentier, N. (2011), "Determinantes de la desigualdad a nivel comunal: el rol de la mujer y la educación", tesis de pregrado, Santiago, Universidad de Chile.

CEPAL (Comisión Económica para América Latina y el Caribe) (2018), La ineficiencia de la desigualdad (LC/SES.37/3-P), Santiago.

_(2017), "Tablas de mortalidad”, Observatorio Demográfico, 2017 (LC/PUB.2017/20-P), Santiago, noviembre.

Cleary, E. (2007), "Chile: entre la imagen de éxito y los fantasmas del subdesarrollo", Polis: Revista Latinoamericana, № 18, Santiago, Centro de Estudios del Desarrollo Regional y Políticas Públicas (CEDER).

Conte, A. (2008), "Desigualdad, polarización y conflicto social", Documentos de Investigación del Programa de Doctorado de Economía Aplicada, Bellaterra, Universidad Autónoma de Barcelona.

Contreras, D. (1999), "Distribución del ingreso en Chile: nueve hechos y algunos mitos", Revista Perspectivas en Política, Economía y Gestión, vol. 2, № 2, Santiago, Universidad de Chile, mayo.

Duclos, J., J. Esteban y D. Ray (2004), "Polarization: concepts, measurement, estimation”, Econometrica, vol. 72, № 6, Sociedad de Econometría, noviembre.

Escobar, M. (1998), "Desviación, desigualdad, polarización: medidas de la diversidad social", Reis: Revista Española de Investigaciones Sociológicas, № 82, Madrid, Centro de Investigaciones Sociológicas (CIS), abril-junio.

Esteban, J. y D. Ray (1994), "On the measurement of polarization", Econometrica, vol. 62, № 4, Sociedad de Econometría, julio.

Gatica, L. y otros (2017), "Predictores psicosociales y psicopolíticos de la justificación de la desigualdad social”, Revista de Psicología, vol. 35, № 1, Lima, Fondo Editorial.

Gradín, C. y M. Rossi (2002), "Polarización y desigualdad salarial en Uruguay, 1986-97”, El Trimestre Económico, vol. 67, № 267, Ciudad de México, Fondo de Cultura Económica, julio-septiembre.

Huesca Reynoso, L. (2003), "Análisis de la polarización del ingreso de los hogares en México durante 1984-2000”, Investigación Económica, vol. 62, № 246, Ciudad de México, Universidad Nacional Autónoma de México, octubre-diciembre.

Jahoda, M. (1958), "Current concepts of positive mental health", Joint Commission on Mental Illness and Health. Monograph Series, № 1, Nueva York, Basic Books.

Keefer, P. y S. Knack (2002), "Polarization, politics and property rights: links between inequality and growth", Public Choice, vol. 111, № 1-2, Springer, marzo.

Kennedy, B. P., I. Kawachi y D. Prothrow-Stith (1996), "Income distribution and mortality: cross sectional ecological study of the Robin Hood index in the United States", British Medical Journal, vol. 312, $\mathrm{N}^{\circ} 7037, \mathrm{BMJ}$, abril.

Martner, R. (2008), "El pacto fiscal y los sistemas tributarios en América Latina: versión preliminar”, Santiago, Instituto Latinoamericano y del Caribe de Planificación Económica y Social (ILPES).

Ministerio de Desarrollo Social (2018), Informe de Desarrollo Social 2018, Santiago [en línea] http://www. desarrollosocialyfamilia.gob.cl/storage/docs/Informe_de_Desarrollo_Social_2018.pdf. (2017), Informe de Desarrollo Social 2017, Santiago [en línea] http://www.desarrollosocialyfamilia.gob. cl/storage/docs/Informe_de_Desarrollo_Social_2017.pdf. (2015), "Resultados Encuesta CASEN 2015" [en línea] http://observatorio.ministeriodesarrollosocial.gob. cl/casen-multidimensional/casen/casen_2015.php.

Nagel, J. (1974), "Inequality and discontent: a nonlinear hypothesis", World Politics, vol. 26, Cambridge University Press, abril.

Navarro Yáñez, C. J. y M. Pérez Yruela (2000), "Calidad de vida y cambio social: de la polarización social a la axiológica en la sociedad andaluza", Revista Internacional de Sociología (RIS), vol. 58, № 26, Consejo Superior de Investigaciones Científicas, mayo-agosto.

OCDE (Organización de Cooperación y Desarrollo Económicos) (2018), OECD Economic Surveys: Chile, París, OECD Publishing, febrero.

(2015), OECD Economic Surveys: Chile, París, OECD Publishing, noviembre.

(2014), Society at a Glance 2014. OECD Social Indicators: The Crisis and its Aftermath, París, OECD Publishing.

Ortiz, I. y M. Cummins (2011), Global Inequality: Beyond the Bottom Billion. A Rapid Review of Income Distribution in 141 Countries, Nueva York, Fondo de las Naciones Unidas para la Infancia (UNICEF), abril.

Osafo Hounkpatin, H. y otros (2015), "Why does income relate to depressive symptoms? Testing the income rank hypothesis longitudinally", Social Indicators Research, vol. 124, № 2, Springer, noviembre. 
Pérez, M. A. (2011), "The emergence of gated communities in the poor periphery: reflections on the new urban segregation and social integration in Santiago, Chile", Berkeley Planning Journal, vol. 24, N 1 , Berkeley, Universidad de California.

Pizarro, R. (2005), "Desigualdad en Chile: desafío económico, ético, y político", Polis: Revista Latinoamericana, NN 10, Santiago, Centro de Estudios del Desarrollo Regional y Políticas Públicas (CEDER).

PNUD (Programa de las Naciones Unidas para el Desarrollo) (2017), Desiguales: orígenes, cambios y desafíos de la brecha social en Chile, Santiago, junio.

Pressacco, C. F. (2009), "El proceso de descentralización regional chileno: avances y "asignaturas pendientes", Papel Político, vol. 14, № 2, Bogotá, Pontificia Universidad Javeriana, julio-diciembre.

Quijada, Y. y otros (2018), "Social inequality and mental health in Chile, Ecuador, and Colombia", Latin American Perspectives, vol. 46, N 6, octubre.

Ramírez, E., A. Tartakowsky y F. Modrego (2009), "La importancia de la desigualdad geográfica en Chile", Documento de Trabajo, N³0, Santiago, Centro Latinoamericano para el Desarrollo Rural (RIMISP).

Raventós, D. (2005), "Propiedad, libertad republicana y Renta Básica de Ciudadanía", Polis: Revista Latinoamericana, N 10, Santiago, Centro de Estudios del Desarrollo Regional y Políticas Públicas (CEDER).

Ruiz-Tagle, J. (1999), "Chile: 40 años de desigualdad de ingresos", Documentos de Trabajo, Santiago, Universidad de Chile.

Ryff, C. D. (1989), "Happiness is everything, or is it? Explorations on the meaning of psychological well-being", Journal of Personality and Social Psychology, vol. 57, № 6, American Psychological Association (APA).

Schatan, J. (2005), "Distribución del ingreso y pobreza en Chile", Polis: Revista Latinoamericana, N 11, Santiago, Centro de Estudios del Desarrollo Regional y Políticas Públicas (CEDER).

Silva Burgos, L. M. (2013), "Cambios observados en la jefatura de hogar en Chile entre los años 1990 y 2009”, Mujeres, su participación económica en la sociedad, G. G. Macías González y E. L. Parada Ruiz (coords.), Guadalajara, Editorial Universitaria/Universidad de Guadalajara.

Solimano, A. y A. Torche (2008), "La distribución del ingreso en Chile 1987-2006: análisis y consideraciones de política", Documentos de Trabajo, N 480, Santiago, Banco Central de Chile, agosto.

Uribe López, M. (2009), "Metas blandas y metas duras en el enfoque de Desarrollo Humano", Polis: Revista Latinoamericana, N²3, Santiago, Centro de Estudios del Desarrollo Regional y Políticas Públicas (CEDER).

Vergara, J. (2011), "Desigualdad, polarización y conflicto en Chile", tesis de maestría, Valencia, Universidad de Valencia.

Villalobos, C. y J. P. Valenzuela (2012), "Polarización y cohesión social del sistema escolar chileno", Revista de Análisis Económico, vol. 27, N², Santiago, Universidad Alberto Hurtado, octubre.

Vivanco Muñoz, R. y otros (2015), "Perpetración y victimización de la violencia en relaciones de parejas en jóvenes que cursan educación superior en la ciudad de Osorno, Chile", Polis: Revista Latinoamericana, N 40, Santiago, Centro de Estudios del Desarrollo Regional y Políticas Públicas (CEDER).

Wilkinson, R. G. (2005), The Impact of Inequality: How to Make Sick Societies Healthier, Nueva York, The New Press, enero.

Wilkinson, R. G. y K. Pickett (2009), Desigualdad: un análisis de la (in)felicidad colectiva, Madrid, Turner.

Wolfson, M. (1997), "Divergent inequalities: theory and empirical results", Review of Income and Wealth, vol. 43, $N^{\circ} 4$, diciembre. 